\title{
Therapeutic efficacy of artemether-lumefantrine and artesunate-amodiaquine for the treatment of uncomplicated Plasmodium falciparum malaria in Mali, 2015-2016
}

\section{Youssouf Diarra}

University of Science, Techniques and Technologies of Bamako

\section{Oumar Koné}

University of Science, Techniques and Technologies of Bamako

\section{Lansana Sangaré}

University of Sciences Techniques and Technologies of Bamako

\section{Lassina Doumbia}

University of Science, Techniques and Technologies of Bamako

\section{Dade Bouye Ben Haidara}

Ministry of Health and Public Health

\section{Mouctar Diallo}

University of Science, Techniques and Technologies of Bamako

\section{Ababacar Maiga}

University of Science, Techniques and Technologies of Bamako

\section{Hammadoun Aly Sango}

University of Science, Techniques and Technologies of Bamako

\section{Halidou Sidibé}

Ministry of Health and Public Hygiene

Jules Mihigo

U.S President's Malaria Initiative, USAID Office, Bamako

\section{Douglas Nace}

Malaria Alert Centers for Disease Control and Prevention, Atlanta

Dragan Ljolje

Malaria Branch, Centers for Disease Control and Prevention, Atlanta

Eldin Talundzic

Malaria Branch, Centers for Disease Control and Prevention, Atlanta

\section{Venkatachalam Udhayakumar}

Malaria Branch, Centers for Disease Control and Prevention, Atlanta

\section{Erin Eckert}

RTI International 


\section{Celia Jane Woodfill}

U.S President's Malaria Initiative, USAID Office, Bamako

\section{Leah F. Moriarty}

Malaria Branch, Centers for Disease Control and Prevention

\section{Pharath Lim}

Medical Care Development International, MD

\section{Donald John Krogstad}

Tulane School of Public Health and Tropical Medicine, New Orleans

\section{Eric S. Halsey}

malaria Branch, Centers for Disease Control and Prevention, U.S President's Malaria Initiative, Atlanta

\section{Naomi W. Lucchi}

Malaria Branch, Centers for Disease Control and Prevention, Atlanta

\section{Ousmane Koita ( $D$ okoita@icermali.org )}

University of Sciences, Tecchniques and Technologies of Bamako https://orcid.org/0000-0001-65353323

\section{Research}

Keywords: malaria, antimalarial resistance, efficacy, Mali, artemether-lumefantrine, artesunateamodiaquine, Pfk13, Pfcrt, Pfmdr-1

Posted Date: September 24th, 2020

DOI: https://doi.org/10.21203/rs.3.rs-81493/v1

License: (1) (1) This work is licensed under a Creative Commons Attribution 4.0 International License. Read Full License 


\section{Abstract \\ Background}

The current first-line treatments for uncomplicated malaria recommended by the National Malaria Control Program in Mali are artemether-lumefantrine (AL) and artesunate-amodiaquine (ASAQ). From 20152016, we conducted an in vivo study to assess the clinical and parasitological responses to AL and ASAQ in Sélingué, Mali.

\section{Methods}

Children between 6 and 59 months of age with uncomplicated Plasmodium falciparum infection and 2,000-200,000 asexual parasites/ $\mu \mathrm{L}$ of blood were enrolled, randomly assigned to either AL or ASAQ, and followed up for 42 days. Uncorrected and PCR-corrected efficacy results at days 28 and 42 were calculated. Known markers of resistance in the Pfk13, Pfmdr1, and Pfcrt genes were assessed using Sanger sequencing.

\section{Results}

A total of 449 patients were enrolled: 225 in the AL group and 224 in the ASAQ group. Uncorrected efficacy at day 28 was $83.4 \%$ (95\% Cl: $78.5-88.4 \%)$ in the AL arm and $93.1 \%(95 \% \mathrm{Cl}: 89.7-96.5 \%)$ in the ASAQ arm. The per protocol PCR-corrected efficacy at day 28 was $91.0 \%(86.0-95.9 \%)$ in the AL arm and $97.1 \%(93.6-100 \%)$ in the ASAQ arm. ASAQ was significantly $(p<0.05)$ better than AL for each of the aforementioned efficacy outcomes. No mutations associated with artemisinin resistance were identified in the Pfk13 gene. Overall, for Pfmdr1, the N86 allele and the NFD haplotype were the most common. The NFD haplotype was significantly more prevalent in the post-treatment than in the pre-treatment isolates in the AL arm $(p<0.01)$ but not in the ASAQ arm. For Pfcrt, the CVIET haplotype was the most common.

\section{Conclusions}

Our findings indicate that both AL and ASAQ remain effective for the treatment of uncomplicated malaria in Sélingué, Mali.

\section{Background}

Integrated strategies to control malaria have led to a decrease in the number of malaria cases and deaths. The incidence rate of malaria declined globally between 2010 and 2018, from 71 to 57 cases per 1000 population at risk [1]. Tracking efficacy and monitoring for parasite resistance helps ensure that antimalarials, critical tools in malaria control, remain effective. 
In 2001, treatment failure using chloroquine was linked to an increase in child mortality in Africa [2]. In 2006, Mali stopped using chloroquine as first-line treatment for uncomplicated malaria and. artemisininbased combination therapy (ACT) was adopted as per the World Health Organization (WHO) recommendation for the treatment of uncomplicated Plasmodium falciparum [3]. The current Mali national malaria case management guidelines recommend the use of either artemether-lumefantrine (AL) or artesunate-amodiaquine (ASAQ) as first-line treatment for uncomplicated malaria. Because amodiaquine is reserved for seasonal malaria chemoprevention, used in combination with sulfadoxinepyrimethamine, ASAQ is not used as a malaria treatment even though it appears as a first-line treatment for uncomplicated malaria in the country's malaria treatment guidelines.

Declining efficacy of ACTs in Southeast Asia (e.g., Cambodia, Thailand) has led to heightened concern for the spread of resistant parasites to other malaria endemic countries $[4,5]$ and the independent emergence of resistant mutants in other malaria endemic countries as was observed in Guyana [6] and Rwanda [7]. Thus, it is necessary to routinely monitor the therapeutic efficacy of ACTs. The monitoring of early changes in $P$. falciparum responses to antimalarial drugs allows for implementation of timely changes in treatment recommendations and policy. Although artemisinin resistance has not yet been widely reported in Africa [1], the intensive use of ACTs worldwide may drive a strong selective pressure for artemisinin resistance.

ACTs are designed such that the artemisinin derivative, with a relatively short half-life, acts rapidly to reduce the parasite burden, while the partner drug, selected for having a longer half-life, continues to eliminate parasites for many days or even weeks after treatment [3]. Thus, there are two concerns to consider during drug efficacy monitoring: 1) inadequate clearance of parasites shortly after drug administration, indicating a possible failure of the artemisinin derivative and 2) recrudescent infection many days or weeks after treatment, which may be associated with failure of the partner drug.

Surveillance of molecular markers associated with the resistance of $P$. falciparum parasites to antimalarial drugs provides an additional way of assessing whether efficacy may be threatened. For instance, certain mutations in the propeller domain of $P$. falciparum kelch 13 (PfK13) gene are associated with artemisinin resistance [8] while polymorphisms in $P$. falciparum multidrug resistance-1 (Pfmdr-1) and $P$. falciparum chloroquine resistance transporter $(P f C r t)$ genes are associated with recurrent parasitemia after treatment with AL and ASAQ [9].

To fulfill the WHO recommendation of performing biannual monitoring of parasite susceptibility to antimalarial drugs in $P$. falciparum endemic areas, we carried out a therapeutic efficacy study of $A L$ and ASAQ for the treatment of uncomplicated malaria. In addition, the study investigated the prevalence of molecular markers associated with resistance and reduced susceptibility to AL and ASAQ.

\section{Methods}

\section{Study sites}


The study was conducted in Sélingué, a commune in the southern part of Mali in the Sikasso region (140 km northwest of Bamako; Fig. 1), where malaria transmission is high due to a relatively long rainy season and the presence of a lake formed by a hydroelectric dam on the Sankarani River. In 2011, malaria parasite prevalence was $42.7 \%$ in children aged five to nine years in Sélingué [10]. Malaria incidence in this area is highest between September and January.

Study design and participants recruitment. The efficacy of AL and ASAQ was assessed in a prospective two-arm study conducted from November 2015 to November 2016, as per the WHO protocol for surveillance of antimalarial drug efficacy [11]. Participants were recruited in the health district of Sélingué among children between 6 and 59 months of age with an axillary temperature $\geq 37.5^{\circ} \mathrm{C}$ or a history of fever during the previous 24 hours, hemoglobin $>5 \mathrm{~g} / \mathrm{dl}$, and uncomplicated $P$. falciparum infection with $2,000-200,000$ asexual parasites/ $\mu \mathrm{L}$ of blood based on microscopy. Children with severe malaria and/or severe anemia (hemoglobin $\leq 5 \mathrm{~g} / \mathrm{dL}$ ) were not included in the study and were referred to the health center for management. Enrolled patients were randomly assigned to either the AL (20/120 mg) or ASAQ (100/270 mg) arm, using a random number generator with PASS11 (pass power analysis and sample size software, NCSS statistical software, https://www.ncss.com/) and randomization was implemented by the research pharmacist. Dosage was weight-based according to manufacturer recommendations. Clinic staff, including physicians and laboratory personnel involved in the study, were blinded to the allocation except for the research pharmacist who provided the treatment. The research pharmacist administered all treatment drugs to each patient on days 0 (day of enrollment), 1 , and 2 . Blood samples were collected on the day of enrollment (pre-treatment); days 1, 2, and 3; and weekly from days 7 to 42 . Participants were followed up with physical and clinical exams after antimalarial administration to evaluate for adverse medication effects or recurrent parasitemia. Enrolled patients were requested to return to the clinic if they experienced any symptoms of malaria during the follow-up period. A blood slide for microscopy and dried blood spots on filter paper were prepared any time a blood sample was collected.

\section{Microscopy}

Thick and thin malaria blood smears were prepared on glass slides and stained with $3 \%$ Giemsa (Sigma; St. Louis, MO) in phosphate buffer ( $\mathrm{pH}$ 7.0). The slide reading was performed by two experienced microscopists at the study sites. If a discrepancy between these two readers exceeded $20 \%$, a third slide read was performed by another senior microscopist and the closest readings averaged for a final parasite count. Parasitemia was estimated according to well-established methods [12].

\section{Sample size estimation:}

A total enrollment of 240 patients per arm would provide adequate power to estimate an efficacy of $95 \%$ with a confidence interval of $\pm 5 \%$, assuming an expected efficacy of $95 \%$ and a maximum loss to followup and withdrawal rate of $20 \%$. This was based on the ability of the drug to clear parasitemia at day 7 or before. 
Clinical monitoring: Each enrolled child received a three-day course of either AL (Coartem, Novartis) or ASAQ (ASAQ-Denk, fixed dose, DENK PHARMA, Munchen, Germany). All doses administered were given under the direct observation of the study pharmacist and both AL and ASAQ were given with a peanutbased paste and milk. All children were observed for a minimum of one hour to monitor for vomiting or other side effects. After the three days of dosing, each enrolled child was followed on days 7, 14, 21, 28, 35, and 42. During each visit, the following were performed or collected: physical exam (weight, height), temperature, thin and thick blood smears, and dried blood spots. Hemoglobin levels were measured at the time of enrollment and on day 42 , at the completion of the study. In cases of recurrent parasitemia, quinine was administered according to the guidelines of the Mali National Malaria Control Program.

Study outcome classification: Treatment responses were classified following the WHO guidelines [11]. Treatment outcomes were classified as either early treatment failure (ETF: day 2 count higher than pretreatment; or day 3 count $\geq 25 \%$ of count on pre-treatment), late clinical failure, late parasitological failure, or adequate clinical and parasitological response (ACPR) as per WHO definitions [3], before and after PCR correction. Children experiencing ETF without danger signs or severe malaria were not offered retreatment. However, children meeting criteria for ETF were not included in the numerator (i.e., as treatment successes) when calculating ACPR.

\section{Molecular analysis}

Genotyping using the merozoite surface proteins 1 and 2 ( $m s p 1$ and $m s p 2$ ), and glutamine-rich protein (glurp) markers, and Sanger sequencing of $P$. falciparum parasites were performed on samples obtained from participants on enrollment day (pre-treatment) and on the day of recurrent parasitemia (posttreatment). Genomic DNA was extracted from all collected samples using the QiAamp mini kit (Qiagen, Valencia, CA USA) following the manufacturer's instructions. Primers designed to amplify three allelic families from block 2 of $m s p 1$ (K1, MAD20 and R033), two allelic families from $m s p 2$ (FC27 and IC/3D7), and the polymorphic region of glurp were used in PCR amplification and analysis as previously described $[13,14]$. Band sizes were scored using an automated Gel Image system (UVP, Upland, CA, USA) and compared across the three markers for paired pre-treatment and day of recurrence samples. For $m s p 1$ and $m s p 2$, bands with lengths within 10 base pairs were considered a match; for glurp, lengths within 50 base pairs were considered a match. If there was at least one matching band in any allelic family for all three markers, the recurrence was classified as a recrudescence (regardless of whether there were additional or missing alleles). If there were no shared alleles for at least one marker, the recurrence was classified as a reinfection. If there were no amplification products resulting in sharp, defined bands in both the pre-treatment and day of recurrence samples for a gene, that gene was not used to distinguish between recrudescence and reinfection, but the aforementioned classification criteria were applied for the genes that were amplified.

Paired pre-treatment and day of recurrent parasitemia samples were assessed for known markers of resistance in the $P f c r t, P f k 13$, and $P f m d r 1$ genes. In addition, all pre-treatment samples were assessed for mutations in the $P f k 13$ and pfmdr1 genes. Fragments of $P f k 13, P f c r t$, and $P f m d r 1$ were amplified by 
nested PCR using previously published primers $[15,16]$. Direct Sanger sequencing of the nested purified PCR products was performed by using a BigDye Terminator v3.1 cycle sequencing kit on an iCycler thermal cycler (Bio-Rad, California, USA). Sequence analysis was performed using Geneouis R7 (Biomatters, Auckland, New Zealand). The Pfcrt codons 72, 74, 75, and 76, Pfk 13 propeller domain (codon positions: 440-600), and Pfmdr1 codons 86, 184, and 1246 were analyzed for single nucleotide polymorphisms (SNPs). The P. falciparum lab strain 3D7 Pfcrt, Pfk13, and Pfmdr1 were used as reference sequences for the analysis. Molecular analyses were performed in collaboration with the U.S. Centers for Disease Control and Prevention (CDC) Malaria Laboratory in Atlanta, USA, as part of the President's Malaria Initiative (PMI)-supported Antimalarial Resistance Monitoring in Africa Network [17].

\section{Statistical analysis}

Treatment responses were classified following the WHO guidelines [11]. Uncorrected efficacy rates were calculated by dividing the number of participants with an ACPR in each arm by the total number of participants. For the PCR-corrected efficacy, per the WHO protocol, new infections identified during followup were not considered as treatment successes or failures and were excluded from the corrected estimations of treatment efficacy [11]. Therefore, patients were censored or excluded from the PCRcorrected analyses if the PCR results indicated that the failure was due to reinfection with $P$. falciparum. Uncorrected and PCR-corrected ACPR for AL and ASAQ were compared at both day 28 and 42 using a chisquare test. The capacity of the two ACTs to clear parasites by day 7 and the post-treatment prophylactic effect of the two treatments was evaluated using a previously described approach by Koita [18]. This approach involved comparing clinical cure rates, a composite of clearance of asexual parasites and fever by day 7. We assessed the post-treatment prophylactic effects of the ACTs by including new infections in the denominator of the uncorrected efficacy estimations. Kaplan Meier estimates were calculated for the uncorrected efficacy, the PCR-corrected efficacy for risk of recrudescence (per WHO guidelines [11]), and the PCR-corrected efficacy for reinfections only (using an approach described in [18]. ). Corresponding survival curves and hazard ratios comparing AL and ASAQ were also generated.

Point mutations in the Pfk13, Pfmdr1, and Pfcrt genes were reported as single or mixed (wild-type and mutant) infections. For samples with mixed infections and SNP variations at multiple sites, each possible haplotype constructed from the observed SNPs was reported for Pfmdr1. In reporting Pfcrt haplotypes in samples with a mixed infection and SNP variations at multiple sites, the wild-type (CVMNK) and most likely mutant type (e.g., CVIET) were reported. The prevalence of the Pfmdr1 mutant alleles and haplotypes was calculated stratifying by the treatment arms (AL and ASAQ) and compared between the pre-treatment samples (samples with ACPR and samples from subjects that later had reinfections) and post-treatment samples (reinfections and recrudescent infections). Likewise, the prevalence of the Pfcrt mutant alleles and haplotypes was compared between pre-treatment and post-treatment samples in the ASAQ arm. For these analyses, pre-treatment samples from participants with recrudescent infections were excluded. Differences between pre-treatment and post-treatment samples were assessed using Fisher's Exact test. The prevalence of wild type versus mutant alleles were compared between groups (e.g., Pfmdr1 N86 versus 86Y). Mixed infections were excluded. To compare haplotypes, the sum of samples 
with the predominant haplotype was compared to the sum of those without that haplotype (e.g., Pfmdr1 haplotype NFD versus all other haplotypes). Statistical significance was defined as $p<0.05$ for all statistical tests. Analyses were performed using Graph Pad Prism version 6.00 for Windows (Graph Pad Software, La Jolla, California, USA) and R (R Foundation for Statistical Computing, Vienna, Austria).

\section{Ethical considerations}

Ethical review and approval was obtained from the Internal Review Board of the Institut National de Recherche en Santé Publique (INRSP, Ministry of Health and Public Hygiene, FWA 00000892). Work performed at the Centers for Disease Control and Prevention was deemed to not constitute engagement in human subject research (CDC, Atlanta, USA; CGH tracking \#2016-012). Parents or guardians of study participants were asked to provide written informed consent.

\section{Results}

\section{Baseline characteristics of study participants}

A total of 3,732 patients were screened for the study and, of these, 449 were enrolled, 225 in the AL group and 224 in the ASAQ group. For parameters such as age, weight, gender, parasitemia, and hemoglobin at the time of enrollment, the two groups were comparable (Table $1 ; p>0.05$ ). Loss to follow-up was comparable $(p>0.05)$ between the two groups at days 28 and 42. The two medications were welltolerated, and no children experienced serious adverse effects from either AL or ASAQ.

\section{Efficacy outcomes}

The ETF rate was $6.5 \%$ in the AL group; this was higher than the rate in the ASAQ group $(1.4 \%$ [p $<0.01])$. We found that both AL and ASAQ were able to clear parasitemia before or on day 7 with $99.5 \%$ and $100 \%$ clearance rates, respectively. A total of 75 patients had recurrent infections during the 42-day follow-up period, 34 of whom had recurrent infections during the first 28 days. The uncorrected efficacy rates on days 28 and 42 were higher in the ASAQ group than in the AL group (Table 2).

The day 28 uncorrected ACPR was 83.4\% (95\% Cl 78.5-88.4\%) for AL and 93.1\% (95\% $\mathrm{Cl} 89.7-96.5 \%)$ for ASAQ, which was significantly different $(p<0.01$; Table 2$)$, a finding also significant at day $42(p<0.01)$. The per-protocol PCR-corrected ACPR rates at day 28 was $91.0 \%$ (95\% $\mathrm{Cl} 86.0-95.9 \%)$ for AL and $97.1 \%$ (95\% $\mathrm{Cl} 93.6-100 \%)$ for ASAQ, which was significantly different between the two groups $(p<0.01)$, a finding also significant at day $42(\mathrm{p}<0.01)$. The majority of recurrent parasitemias during the 42 -day follow-up period were classified as new infections rather than recrudescent infections, $88.6 \%$ (39/44) for AL and $87.1 \%(27 / 31)$ for ASAQ, most of which occurred after 28 days.

The uncorrected day 28 Kaplan Meier estimates were 83.6\% (95\% Cl 78.8-88.6\%; Table 3) for AL and 93.2\% (95\% Cl 89.9-96.6\%) for ASAQ. The PCR-corrected day 28 Kaplan Meier estimates were 91.9\% (95\% Cl 88.3-95.5\%) for AL and 97.3\% (95\% Cl 95.1-99.4\%) for ASAQ. The day 28 cumulative risk of 
treatment failure was statistically significantly higher for the AL arm than the ASAQ arm for risk of reinfections plus recrudescences (uncorrected; hazard ratio 2.58), recrudescences only (PCR-corrected; hazard ratio 3.12), and reinfections only (hazard ratio 2.59; Fig. 2, Table 3).

Results of genotyping using the $m s p 1, m s p 2$, and glurp genes can be found in Supplemental Table 1 .

\section{Molecular markers of drug resistance}

A total of $296 / 386(76.6 \%)$ samples were successfully assayed for polymorphisms in the $P f k 13$ gene (248 pre-treatment and 48 post-treatment samples). We did not observe any of the known mutations associated with artemisinin resistance in the $P f k 13$ gene. However, we observed synonymous polymorphisms in some pretreatment samples at codons: T478T, Y493Y, K503K, and Q613Q, all in one sample each. We found the synonymous polymorphism C469C in two samples and the A578S mutant allele was observed in two samples (pre-treatment and day 28) collected from the same patient.

A total of 388 samples were sequenced for the $p f m d r 1$ gene (314 pre-treatment and 74 post-treatment samples). Overall, the wildtype N86 allele was found in majority of the successfully sequenced pretreatment $(200 / 246,81.3 \%)$ and post-treatment $(57 / 63,90.5 \%)$ isolates. Similarly, the overall prevalence of the $184 \mathrm{~F}$ allele was high in pre-treatment $(168 / 284,59.2 \%)$ and in the post-treatment isolates $(47 / 62$, $75.8 \%$; Table 4). The NFD haplotype was overrepresented in the post-treatment compared to the pretreatment isolates in the AL arm $(77.1 \%$ vs $59.7 \%, p<0.01)$ but not in the ASAQ arm $(70.8 \%$ vs $61.7 \%, p=$ 1.00). A similar trend was observed for the prevalence of the N86 allele, which was higher in the posttreatment $(97.2 \%)$ than the pre-treatment $(81.7 \%)$ isolates in the $A L$ arm, although the difference was not statistically different $(p=0.195)$. The prevalence of the N86 allele was similar in the pre-treatment $(80.8 \%)$ and post-treatment $(81.5 \%)$ isolates in the ASAQ arm $(p=0.766)$ (Table 4$)$.

The Pfcrt gene was investigated in pre-treatment and post-treatment matched pairs of samples in the ASAQ arm of the study. A total of 60/62 (97\%) samples were successfully sequenced. Mutations were observed in the M74I, N75E and K76T codons. The pfcrt 76T allele, associated with amodiaquine resistance, was found in $57.7 \%$ of pre-treatment and $40.0 \%$ of post-treatment isolates, $(p=0.018$; Table 5$)$. Mixed infections with $76 \mathrm{~K} / \mathrm{T}$ were found in $7(26.9 \%)$ and $2(6.7 \%)$ of pre-treatment and post-treatment isolates, respectively. All isolates sequenced harbored either the mutant CVIET or wild-type CVMNK haplotypes.

\section{Discussion And Conclusion}

Declining susceptibility of $P$. falciparum parasites to artemisinin derivatives and partner drugs in Southeast Asia, along with the threat of an introduction or the development of these resistant parasites in Africa, requires active monitoring of treatment efficacy and of genetic markers associated with antimalarial drug resistance in malaria endemic areas. In this study conducted in Mali, both AL and ASAQ were efficacious. 
Even though this study investigated efficacy results through 42 days, WHO recommends reporting ACPR for AL and ASAQ at day 28 , with $90 \%$ being the threshold where a change in first-line treatment should be considered [11]. The day 28 PCR-corrected efficacies for both AL (91.0\%) and ASAQ (97.1\%) were above this threshold. However, when accounting for a 95\% confidence interval, the 86.0-95.9\% PCR-corrected efficacy range of $A L$ indicates that continued frequent efficacy monitoring of this drug is warranted in Mali.

A handful of studies over the past decade have investigated the efficacy of AL in Mali, and reported PCRcorrected efficacies greater than 98\%. These include: a 2010-2014 study in Sotuba, Kolle, and Banambani [20]; a 2012-2014 study in Dioro [21]; and a 2013-2015 study in Doneguebougou and Torodo [22]. Although two of these studies [20,22] considered reinfections as treatment successes in their calculation of PCR-corrected efficacy (an approach not consistent with WHO guidelines), the efficacy results remained above $97 \%$ when recalculating efficacy using the WHO-endorsed approach of eliminating reinfections from the calculation. Efficacy results above $90 \%$ were reported in another Mali study [23], conducted in 2013-2015, although this study reported combined results in children from another country, Niger, in addition to Koulikoro, Mali. Similar to the AL day 28 uncorrected efficacy of $83.4 \%$ in our study, the two other Mali-exclusive studies that examined day 28 uncorrected efficacy reported results of $83.8 \%$ and $84.5 \%[20,22]$.

The most recent publication on the efficacy of ASAQ in Mali is from a study conducted 2005-2007 in Bougoula-Hameau [24]. This study reported a $98.7 \%$ day 28 PCR-corrected efficacy (the rate was $98.3 \%$ when recalculated per WHO methodology) and a 78.5\% day 28 uncorrected efficacy. Other ACTs endorsed by WHO for treatment of uncomplicated malaria have been shown to have PCR-corrected efficacies greater than 90\% in Mali, including dihydroartemisinin-piperaquine (DP) [22], artesunate-pyronaridine [25], and artesunate plus sulfadoxine-pyrimethamine [20].

Although there were 14 and 3 ETFs in the AL and ASAQ arms, respectively, nearly all subjects cleared parasitemia by day 7 implying that both treatment are able to clear parasites early in the infection.. Compared with AL, ASAQ's higher day 28 and 42 uncorrected efficacies may be a result of the longer halflife of amodiaquine compared with lumefantrine [3]. For both drug arms, a majority of reinfections occurred between days 29-42 compared with days 7-28; ACT containing a partner drug with a longer half-life, such as piperaquine in DP [3] may confer an even longer window of protection against a future infection.

No PfK13 mutations associated with artemisinin resistance were found in any of the parasites investigated, consistent with other studies carried out in Africa where malaria parasites are largely wildtype in the $P f k 13$ propeller domain and remain sensitive to artemisinin derivatives $[8,26]$. However, other PfK13 polymorphisms were detected at six positions. The A578S polymorphism has been found throughout Africa and so far has not been associated with resistance to artemisinin derivatives but appears as a common polymorphism observed in African parasites [8, 26, 27]. A mutation at position 493 (Y493H) is one of the PfK13 mutations associated with artemisinin resistance [8]; however, we found a 
silent mutation at this codon, Y493Y (TAC $\rightarrow$ TAT), in one sample. While it is possible this is a transitory mutation that could lead to the artemisinin resistant allele $\mathrm{Y} 493 \mathrm{H}$, no data currently exists to support this notion.

Equally important in the efficacy of an ACT is the partner drug. While delayed clearance (within 3 days post treatment) of parasites is associated with resistance to the artemisinin component, parasite recrudescence may occur when resistance to the partner drug exists, leading to an inadequate clinical and parasitological response. It is therefore imperative that resistance markers to partner drugs are evaluated. Point mutations in the pfcrt and pfmdr1 genes are associated with decreased sensitivity to lumefantrine and amodiaquine [9]. In particular, the prevalence of the Pfmdr1 N86 allele and NFD haplotype have been shown to be associated with reduced susceptibility to lumefantrine in some studies. In agreement with previous studies conducted in other parts of Africa [16, 28, 29], including Mali [30], we observed a higher prevalence of the NFD haplotype in the post-treatment isolates in the AL arm compared to pre-treatment isolates. We did not observe this in the ASAQ arm, suggesting that parasites harboring the NFD haplotypes are likely to be more tolerant to AL treatment, consistent with previous observations. Overall, the prevalence of the N86 allele was more than $80 \%$ in the pre-treatment isolates in our study, similar to what was recently reported in two study sites in Mali, Dangassa and Nioro-du-Sahel [30]. In addition, a majority (59.2\%) of the baseline isolates in our study harbored the $184 \mathrm{~F}$ allele, which can be contrasted with previous reports from Dangassa (39.9\%) and Nioro-du-Sahel (48.2\%). The significance of the high prevalence of N86 is unclear and may simply reflect the withdrawal of chloroquine drug pressure that selected for the $86 \mathrm{Y}$ polymorphism as previously indicated [31] and/or a true reflection of the continued AL use in Mali. The reduced prevalence of the $86 \mathrm{Y}$ allele in Mali, however, provides further support for the use of amodiaquine, a drug combined with sulfadoxine-pyrimethamine and used for seasonal malaria chemoprevention in children under five years in Mali.

The Pfcrt 76T mutation has also been shown to play a role in amodiaquine resistance in several studies in Africa [9]. Prior studies have demonstrated the selection of this allele after amodiaquine monotherapy [32] or ASAQ treatment $[9,28,33,34]$. We observed a lower prevalence of the Pfcrt 76T allele in the posttreatment compared to pre-treatment isolates in our study, in contrast to previous reports. These unexpected findings can be explained by the fact that majority (27) of the post-treatment isolates in our study were reinfection isolates and only 4 recrudescent isolates (among which 3 had the 76T mutation). The withdrawal of chloroquine for the treatment of malaria was followed by a steady decline of the prevalence of the Pfcrt 76T allele in many African countries [35]. However, this decline has varied by region, with West Africa still showing a steady prevalence of 58.3\% [35]. The baseline prevalence of this allele in our study was $57.7 \%$, similar to observations reported by others [30] who found equally high prevalence of this allele in Dangassa (64.3\%) and Nioro-du-Sahel (42.5\%). Whether this is a result of increased use of amodiaquine is unknown and additional surveillance studies are required.

Although we investigated molecular markers in post-treatment samples (by combining samples with reinfections and recrudescent infection), association between observed parasite genotypes and recrudescent infection was not possible due to the small number of recrudescent infections $(n=9)$ which, 
when analyzed alone, did not provide sufficient power. Nonetheless, monitoring of molecular markers of resistance to partner drugs during a therapeutic efficacy study is becoming increasingly feasible and provides a cost-effective tool for the early detection of decreases in parasite susceptibility to the drugs.

This study demonstrated that day 28 PCR-corrected efficacy of both AL and ASAQ for uncomplicated malaria exceeded $90 \%$, a WHO-recommended cut-off below which alternative treatments should be considered. Both uncorrected and PCR-corrected results suggested that ASAQ may be more efficacious, although the study was not powered to provide a comparison; however, this ACT is not employed routinely as a first-line malaria treatment because amodiaquine is paired with sulfadoxine-pyrimethamine and used throughout the country for seasonal malaria chemoprevention. Future studies in Mali should continue to monitor the efficacy of the current first-line ACTs and consideration should also be given to evaluating ACTs, such as DP, with longer acting partner drugs.

\section{Declarations}

\section{Competing interests}

The authors have no competing interests.

\section{Statement regarding data and materials availability}

the datasets will be available on the WWARN (https://www.wwarn.org)

\section{Consent for publication}

All the authors have given their consent for publication

\section{Author's contributions}

OAK, DJK, JM, CJW and ESH have conceived the study protocol. YD, OK, LS, LD, DBBH, MD, AM, HAS, HS, PL have collected the data in the field and performed the genotyping of $P$. falciparum isolates. YD, DN, DL, ET, NWL have performed and analyzed the sequencing data. ESH, LFM, JM, NWL, CJW, VU have written the manuscript. All authors have reviewed the manuscript.

\section{Acknowledgments}

The authors would like to thank the National Malaria Control Program of Mali, all study team members in Sélingué, and children's parents and relatives. We thank the USP/QM for managing funds under USAID Cooperative Agreement No. GHS-A-00-09-00003-00, Promoting the Quality of Medicines Program (FAA-1602) and ICEMR NIAID U19 AI 089696. The authors thank Kelsey Rondini for her assistance in creating the molecular markers of resistance findings table.

The findings and conclusions in this report are those of the author(s) and do not necessarily represent the official position of the Centers for Disease Control and Prevention. 


\section{References}

1. World malaria report 2019. Geneva: World Health Organization; 2019.

2. Trape J. The public health impact of chloroquine resistance in Africa. The American Journal of Tropical Medicine and Hygiene. 2001 Jan 1;64(1_suppl):12-7.

3. World Health Organization. Guidelines for the treatment of malaria. Geneva: World Health Organization; 2015.

4. Dondorp AM, Nosten F, Yi P, Das D, Phyo AP, Tarning J, et al. Artemisinin Resistance in Plasmodium falciparum Malaria. New England Journal of Medicine. 2009 Jul 30;361(5):455-67.

5. Ashley EA, Dhorda M, Fairhurst RM, Amaratunga C, Lim P, Suon S, et al. Spread of Artemisinin Resistance in Plasmodium falciparum Malaria [Internet]. http://dx.doi.org/10.1056/NEJMoa1314981. Massachusetts Medical Society; 2014 [cited 2020 Mar 27]. Available from: https://www.nejm.org/doi/10.1056/NEJMoa1314981

6. Chenet SM, Okoth SA, Kelley J, Lucchi N, Huber CS, Vreden S, et al. Molecular Profile of Malaria Drug Resistance Markers of Plasmodium falciparum in Suriname. Antimicrob Agents Chemother [Internet]. 2017 Jun 27 [cited 2020 Sep 9];61(7). Available from: https://www.ncbi.nlm.nih.gov/pmc/articles/PMC5487647/

7. Uwimana A, Legrand E, Stokes BH, Ndikumana J-LM, Warsame M, Umulisa N, et al. Emergence and clonal expansion of in vitro artemisinin-resistant Plasmodium falciparum kelch13 R561H mutant parasites in Rwanda. Nature Medicine. 2020 Aug 3;1-7.

8. WHO | Status report on artemisinin resistance and ACT efficacy (August 2018) [Internet]. WHO. World Health Organization; [cited 2020 May 25]. Available from: http://www.who.int/malaria/publications/atoz/artemisinin-resistance-august2018/en/

9. Venkatesan M, Gadalla NB, Stepniewska K, Dahal P, Nsanzabana C, Moriera C, et al. Polymorphisms in Plasmodium falciparum Chloroquine Resistance Transporter and Multidrug Resistance 1 Genes: Parasite Risk Factors That Affect Treatment Outcomes for P. falciparum Malaria After ArtemetherLumefantrine and Artesunate-Amodiaquine. The American Journal of Tropical Medicine and Hygiene. 2014 Oct 1;91(4):833-43.

10. Touré M, Sanogo D, Dembele S, Diawara SI, Oppfeldt K, Schiøler KL, et al. Seasonality and shift in age-specific malaria prevalence and incidence in Binko and Carrière villages close to the lake in Selingué, Mali. Malar J. 2016 Dec;15(1):219.

11. WHO. Methods for surveillance of antimalarial drug efficacy. World Health Organization. 2009;

12. Payne D. Use and limitations of light microscopy for diagnosing malaria at the primary health care level. :6.

13. World Health Organization. Methods and techniques for clinical trials on antimalarial drug efficacy: genotyping to identify parasite populations [Internet]. Geneva: World Health Organization; 2007. Available from: http://www.who.int/iris/handle/10665/43824 
14. Manual size estimation of msp1, msp2 and glurp PCR products on gel [Internet]. Worldwide Antimalarial Resistance Network. 2014 [cited 2020 Jun 9]. Available from: https://www.wwarn.org/tools-resources/procedures/manual-size-estimation-msp1-msp2-and-glurppcr-products-gel

15. Talundzic E, Okoth SA, Congpuong K, Plucinski MM, Morton L, Goldman IF, et al. Selection and Spread of Artemisinin-Resistant Alleles in Thailand Prior to the Global Artemisinin Resistance Containment Campaign. Fairhurst RM, editor. PLoS Pathog. 2015 Apr 2;11(4):e1004789.

16. Malmberg M, Ngasala B, Ferreira PE, Larsson E, Jovel I, Hjalmarsson A, et al. Temporal trends of molecular markers associated with artemether-lumefantrine tolerance/resistance in Bagamoyo district, Tanzania. Malar J. 2013 Dec;12(1):103.

17. Halsey ES, Venkatesan M, Plucinski MM, Talundzic E, Lucchi NW, Zhou Z, et al. Capacity Development through the US President's Malaria Initiative-Supported Antimalarial Resistance Monitoring in Africa Network. Emerg Infect Dis [Internet]. 2017 Dec [cited 2020 Feb 11];23(13). Available from: http://wwwnc.cdc.gov/eid/article/23/13/17-0366_article.htm

18. Koita OA, Sangaré L, Miller HD, Sissako A, Coulibaly M, Thompson TA, et al. AQ-13, an investigational antimalarial, versus artemether plus lumefantrine for the treatment of uncomplicated Plasmodium falciparum malaria: a randomised, phase 2, non-inferiority clinical trial. The Lancet Infectious Diseases. 2017;

19. Tinto H, Diallo S, Zongo I, Guiraud I, Valea I, Kazienga A, et al. Effectiveness of artesunateamodiaquine $v s$. artemether-lumefantrine for the treatment of uncomplicated falciparum malaria in Nanoro, Burkina Faso: a non-inferiority randomised trial. Trop Med Int Health. 2014 Apr;19(4):46975.

20. Ringwald P, Guindo CO, Niaré K, Benoit-Vical F, Coulibaly CO, Djimdé AA, et al. In Vivo Efficacy and Parasite Clearance of Artesunate + Sulfadoxine-Pyrimethamine Versus Artemether-Lumefantrine in Mali. The American Journal of Tropical Medicine and Hygiene. 2016 Mar 2;94(3):634-9.

21. Dieye B, Affara M, Sangare L, Joof F, Ndiaye YD, Gomis JF, et al. West Africa International Centers of Excellence for Malaria Research: Drug Resistance Patterns to Artemether-Lumefantrine in Senegal, Mali, and The Gambia. The American Journal of Tropical Medicine and Hygiene. 2016 Nov 2;95(5):1054-60.

22. Dama S, Niangaly H, Djimde M, Sagara I, Guindo CO, Zeguime A, et al. A randomized trial of dihydroartemisinin-piperaquine versus artemether-lumefantrine for treatment of uncomplicated Plasmodium falciparum malaria in Mali. Malaria Journal. 2018 Oct 5;17(1):347.

23. Denoeud-Ndam L, Dicko A, Baudin E, Guindo O, Grandesso F, Diawara H, et al. Efficacy of artemetherlumefantrine in relation to drug exposure in children with and without severe acute malnutrition: an open comparative intervention study in Mali and Niger. BMC Med. 2016 24;14(1):167.

24. Sagara I, Fofana B, Gaudart J, Sidibe B, Togo A, Toure S, et al. Repeated Artemisinin-Based Combination Therapies in a Malaria Hyperendemic Area of Mali: Efficacy, Safety, and Public Health Impact. Am J Trop Med Hyg. 2012 Jul 1;87(1):50-6. 
25. Sagara I, Beavogui AH, Zongo I, Soulama I, Borghini-Fuhrer I, Fofana B, et al. Safety and efficacy of re-treatments with pyronaridine-artesunate in African patients with malaria: a substudy of the WANECAM randomised trial. Lancet Infect Dis. 2016 Feb;16(2):189-98.

26. Taylor SM, Parobek CM, De Conti DK, Kayentao K, Coulibaly SO, Greenwood BM, et al. Absence of putative artemisinin resistance mutations among Plasmodium falciparum in sub-Saharan Africa: A molecular epidemiologic study. Journal of Infectious Diseases. 2015;

27. Ménard D, Khim N, Beghain J, Adegnika AA, Shafiul-Alam M, Amodu O, et al. A Worldwide Map of Plasmodium falciparum K13-Propeller Polymorphisms. N Engl J Med. 2016 Jun 23;374(25):245364.

28. Otienoburu SD, Maïga-Ascofaré O, Schramm B, Jullien V, Jones JJ, Zolia YM, et al. Selection of Plasmodium falciparum pfcrt and pfmdr1 polymorphisms after treatment with artesunateamodiaquine fixed dose combination or artemether-lumefantrine in Liberia. Malar J. 2016 Dec;15(1):452.

29. Happi CT, Gbotosho GO, Folarin OA, Sowunmi A, Hudson T, O'Neil M, et al. Selection of Plasmodium falciparum Multidrug Resistance Gene 1 Alleles in Asexual Stages and Gametocytes by ArtemetherLumefantrine in Nigerian Children with Uncomplicated Falciparum Malaria. AAC. 2009 Mar;53(3):888-95.

30. Diakité SAS, Traoré K, Sanogo I, Clark TG, Campino S, Sangaré M, et al. A comprehensive analysis of drug resistance molecular markers and Plasmodium falciparum genetic diversity in two malaria endemic sites in Mali. Malar J. 2019 Dec;18(1):361.

31. Lekana-Douki JB, Boutamba SDD, Zatra R, Edou SEZ, Ekomy H, Bisvigou U, et al. Increased prevalence of the Plasmodium falciparum Pfmdr1 $86 \mathrm{~N}$ genotype among field isolates from Franceville, Gabon after replacement of chloroquine by artemether-lumefantrine and artesunatemefloquine. Infection, Genetics and Evolution. 2011 Mar;11(2):512-7.

32. Tekete M, Djimde AA, Beavogui AH, Maiga $H$, Sagara I, Fofana B, et al. Efficacy of chloroquine, amodiaquine and sulphadoxine-pyrimethamine for the treatment of uncomplicated falciparum malaria: revisiting molecular markers in an area of emerging AQ and SP resistance in Mali. Malar J. 2009;8(1):34.

33. Djimdé AA, Fofana B, Sagara I, Sidibe B, Toure S, Dembele D, et al. Efficacy, safety, and selection of molecular markers of drug resistance by two ACTs in Mali. Am J Trop Med Hyg. 2008 Mar;78(3):455-61.

34. Sondo P, Derra K, Diallo Nakanabo S, Tarnagda Z, Kazienga A, Zampa O, et al. ArtesunateAmodiaquine and Artemether-Lumefantrine Therapies and Selection of Pfcrt and Pfmdr1 Alleles in Nanoro, Burkina Faso. Woodrow CJ, editor. PLoS ONE. 2016 Mar 31;11(3):e0151565.

35. Ocan M, Akena D, Nsobya S, Kamya MR, Senono R, Kinengyere AA, et al. Persistence of chloroquine resistance alleles in malaria endemic countries: a systematic review of burden and risk factors. Malar J. 2019 Dec;18(1):76. 


\section{Tables}

Table 1: Participant characteristics at enrollment and follow-up, Mali 2015-2016.

\begin{tabular}{|c|c|c|c|c|}
\hline Study arm & $\mathrm{AL}$ & ASAQ & Total & $\begin{array}{l}\text { p- } \\
\text { value }\end{array}$ \\
\hline \multicolumn{5}{|l|}{ Participant characteristics at baseline } \\
\hline Enrolled & 225 & 224 & & \\
\hline Median age, month (range) & $36.0(7-59)$ & $36.0(6-59)$ & & 0.970 \\
\hline Median weight, kg (range) & $13.0(9-21)$ & $13.3(9-20)$ & & 0.872 \\
\hline Number male/female & $126 / 99$ & $111 / 113$ & & 0.083 \\
\hline $\begin{array}{l}\text { Median pre-treatment parasitemia } \\
\text { (parasite/ } \mu \mathrm{L})\end{array}$ & $\begin{array}{l}11750(2000- \\
199800)\end{array}$ & $\begin{array}{l}11825(2000- \\
197500)\end{array}$ & & 0.659 \\
\hline $\begin{array}{l}\text { Median pre-treatment hemoglobin } \\
(\mathrm{g} / \mathrm{dL})\end{array}$ & $9.4(5-13)$ & $9.0(5-13)$ & & 0.161 \\
\hline
\end{tabular}

AL: artemether-lumefantrine

ASAQ: artesunate-amodiaquine

Table 2: Uncorrected and PCR-corrected treatment outcomes at days 28 and 42 for artemetherlumefantrine (AL) and artesunate-amodiaquine (ASAQ), Mali 2015-2016. 


\begin{tabular}{|c|c|c|c|c|c|c|c|c|c|}
\hline $\begin{array}{l}\text { Uncorrected } \\
\text { treatment } \\
\text { outcomes - } \\
\text { day } 28\end{array}$ & $\mathrm{AL}$ & & $95 \% \mathrm{Cl}$ & & ASAQ & & $95 \% \mathrm{Cl}$ & & $\begin{array}{l}\text { p- } \\
\text { value }\end{array}$ \\
\hline $\begin{array}{l}\text { Number } \\
\text { reaching an } \\
\text { endpoint }\end{array}$ & 217 & & Lower & Upper & 217 & & Lower & Upper & \\
\hline $\begin{array}{l}\text { Early } \\
\text { treatment } \\
\text { failure }\end{array}$ & 14 & $6.5 \%$ & $3.2 \%$ & $9.7 \%$ & 3 & $1.4 \%$ & $0.0 \%$ & $2.9 \%$ & $0.006^{\dagger}$ \\
\hline $\begin{array}{l}\text { Late clinical } \\
\text { failure }\end{array}$ & 10 & $4.6 \%$ & & & 5 & $2.3 \%$ & & & \\
\hline $\begin{array}{l}\text { Late } \\
\text { parasitological } \\
\text { failure }\end{array}$ & 12 & $5.5 \%$ & & & 7 & $3.2 \%$ & & & \\
\hline $\begin{array}{l}\text { Adequate } \\
\text { clinical and } \\
\text { parasitological } \\
\text { response at } \\
\text { day } 28\end{array}$ & 181 & $83.4 \%$ & $78.5 \%$ & $88.4 \%$ & 202 & $93.1 \%$ & $89.7 \%$ & $96.5 \%$ & $0.002^{\dagger}$ \\
\hline $\begin{array}{l}\text { Uncorrected } \\
\text { treatment } \\
\text { outcomes - } \\
\text { day } 42\end{array}$ & & & & & & & & & - \\
\hline $\begin{array}{l}\text { Number } \\
\text { reaching an } \\
\text { endpoint }\end{array}$ & 212 & & & & 214 & & & & \\
\hline $\begin{array}{l}\text { Early } \\
\text { treatment } \\
\text { failure }\end{array}$ & 14 & $6.6 \%$ & $3.3 \%$ & $9.9 \%$ & 3 & $1.4 \%$ & $0.0 \%$ & $3.0 \%$ & $0.006^{\dagger}$ \\
\hline $\begin{array}{l}\text { Late clinical } \\
\text { failure }\end{array}$ & 15 & $7.1 \%$ & & & 9 & $4.2 \%$ & & & \\
\hline $\begin{array}{l}\text { Late } \\
\text { parasitological } \\
\text { failure }\end{array}$ & 29 & $13.7 \%$ & & & 22 & $10.3 \%$ & & & \\
\hline $\begin{array}{l}\text { Adequate } \\
\text { clinical and } \\
\text { parasitological } \\
\text { response at } \\
\text { day } 42\end{array}$ & 154 & $72.6 \%$ & $66.6 \%$ & $78.6 \%$ & 180 & $84.1 \%$ & $78.3 \%$ & $89.9 \%$ & $0.004^{\dagger}$ \\
\hline $\begin{array}{l}\text { PCR corrected } \\
\text { treatment } \\
\text { outcomes - } \\
\text { day } 28\end{array}$ & & & & & & & & & - \\
\hline $\begin{array}{l}\text { Number } \\
\text { reaching an }\end{array}$ & 217 & & & & 217 & & & & \\
\hline
\end{tabular}


endpoint

\begin{tabular}{|c|c|c|c|c|c|c|c|c|c|}
\hline $\begin{array}{l}\text { Early } \\
\text { treatment } \\
\text { failure }\end{array}$ & 14 & $6.5 \%$ & $3.2 \%$ & $9.7 \%$ & 3 & $1.4 \%$ & $0.0 \%$ & $2.9 \%$ & $0.006^{\dagger}$ \\
\hline $\begin{array}{l}\text { Recrudescent } \\
\text { cases }\end{array}$ & 4 & $1.8 \%$ & & & 3 & $1.4 \%$ & & & \\
\hline $\begin{array}{l}\text { Total new } \\
\text { infections }\end{array}$ & 18 & $8.3 \%$ & & & 9 & & & & \\
\hline Day 7-14 & 1 & & & & 2 & & & & \\
\hline Day 15-21 & 10 & & & & 1 & & & & \\
\hline Day 22-28 & 7 & & & & 6 & & & & \\
\hline $\begin{array}{l}\text { Adequate } \\
\text { clinical and } \\
\text { parasitological } \\
\text { response at } \\
\text { day } 28\end{array}$ & 181 & $91.0 \%$ & $86.0 \%$ & $95.9 \%$ & 202 & $97.1 \%$ & $93.6 \%$ & $100.0 \%$ & $0.008^{\dagger}$ \\
\hline
\end{tabular}

\section{PCR corrected \\ treatment \\ outcomes - \\ day 42}

Number

212

214

reaching an

endpoint

\begin{tabular}{|c|c|c|c|c|c|c|c|c|}
\hline $\begin{array}{l}\text { Early } \\
\text { treatment } \\
\text { failure }\end{array}$ & 14 & $6.6 \%$ & $3.3 \%$ & $9.9 \%$ & 3 & $1.4 \%$ & $0.0 \%$ & $3.0 \%$ \\
\hline
\end{tabular}

$\begin{array}{lllll}\text { Recrudescent } & 5 & 2.4 \% & 4 & 1.9 \%\end{array}$

cases

$\begin{array}{lllll}\text { Total new } & 39 & 18.4 \% & 27 & 12.6 \%\end{array}$

infections

Day 7-14 112

$\begin{array}{lll}\text { Day } 15-21 & 10 & 1\end{array}$

$\begin{array}{lll}\text { Day } 22-28 & 7 & 6\end{array}$

$\begin{array}{lll}\text { Day 29-35 } 16 & 12\end{array}$

Day 36-42 5

$\begin{array}{llllllllll}\text { Adequate } & 154 & 89.0 \% & 83.0 \% & 95.0 \% & 180 & 96.3 \% & 91.0 \% & 100.0 \% & 0.008^{\dagger}\end{array}$ clinical and parasitological response at day 42 
$\mathrm{AL}$ : artemether lumefantrine

ASAQ: artesunate-amodiaquine

${ }^{\dagger} \mathrm{p}<0.05$

Table 3: Kaplan Meier efficacy estimates and hazard ratios between AL and ASAQ, therapeutic efficacy monitoring, Mali, 2015-2016.

\begin{tabular}{|c|c|c|c|c|c|c|c|c|}
\hline & $\mathrm{AL}$ & $95 \% \mathrm{Cl}$ & & ASAQ & $95 \% \mathrm{Cl}$ & & $\begin{array}{l}\text { Hazard } \\
\text { Ratio }\end{array}$ & $\begin{array}{l}\mathrm{p}- \\
\text { value }\end{array}$ \\
\hline \multicolumn{9}{|l|}{ Uncorrected } \\
\hline $\begin{array}{l}\text { Kaplan Meier } \\
\text { estimate - day } 28\end{array}$ & $83.6 \%$ & $78.8 \%$ & $88.6 \%$ & $93.2 \%$ & $89.9 \%$ & $96.6 \%$ & $\begin{array}{l}2.58 \\
(1.4 \\
4.7)\end{array}$ & $0.002^{+}$ \\
\hline $\begin{array}{l}\text { Kaplan Meier } \\
\text { estimate - day } 42\end{array}$ & $73.1 \%$ & $67.4 \%$ & $79.3 \%$ & $84.4 \%$ & $79.7 \%$ & $89.4 \%$ & $\begin{array}{l}1.9 \\
(1.2, \\
2.9)\end{array}$ & $0.003^{\dagger}$ \\
\hline \multicolumn{9}{|l|}{ PCR-corrected } \\
\hline $\begin{array}{l}\text { Kaplan Meier } \\
\text { estimate - day } 28\end{array}$ & $91.9 \%$ & $88.3 \%$ & $95.5 \%$ & $97.3 \%$ & $95.1 \%$ & $99.4 \%$ & $\begin{array}{l}3.12 \\
(1.2, \\
8.9)\end{array}$ & $0.016^{\dagger}$ \\
\hline $\begin{array}{l}\text { Kaplan Meier } \\
\text { estimate - day } 42\end{array}$ & $91.3 \%$ & $87.6 \%$ & $95.1 \%$ & 96.8 & 94.4 & 99.2 & $\begin{array}{l}2.9 \\
(1.2, \\
6.8)\end{array}$ & $0.018^{\dagger}$ \\
\hline \multicolumn{9}{|l|}{ Reinfections } \\
\hline $\begin{array}{l}\text { Kaplan Meier } \\
\text { estimate - day } 28\end{array}$ & $90.9 \%$ & $86.9 \%$ & $95.0 \%$ & $95.7 \%$ & $93.1 \%$ & $98.5 \%$ & $\begin{array}{l}2.59 \\
(1.42 \\
4.73)\end{array}$ & $0.002^{\dagger}$ \\
\hline $\begin{array}{l}\text { Kaplan Meier } \\
\text { estimate - day } 42\end{array}$ & $80.0 \%$ & $74.6 \%$ & $85.8 \%$ & $87.2 \%$ & $82.8 \%$ & $91.8 \%$ & $\begin{array}{l}1.65 \\
(1.0 \\
2.7)^{\prime}\end{array}$ & $0.045^{\dagger}$ \\
\hline
\end{tabular}

AL: artemether lumefantrine

ASAQ: artesunate-amodiaquine

${ }^{\dagger} p<0.05$

Table 4: Prevalence of Pfmdr1 polymorphisms in pre-treatment and post-treatment samples stratified by treatment arms, therapeutic efficacy monitoring, Mali, 2015-2016. 
AL arm

\section{Pre- \\ Post-} treatment $n$, (\%) treatment $\mathrm{n}, \quad$ value $^{\star}$ (\%)
ASAQ arm

$\begin{array}{lll}\text { Pre- } & \begin{array}{l}\text { Post- } \\ \text { treatment } n,\end{array} & \text { p- } \\ \text { treatment } n, & \text { value* }\end{array}$ (\%)

\section{Pfmdr1 point}

mutations

\begin{tabular}{|c|c|c|c|c|c|c|}
\hline $\begin{array}{l}\text { Pfmdr1 codon } \\
86\end{array}$ & $n=126$ & $n=36$ & & $n=120$ & $n=27$ & \\
\hline N86 & $103(81.7)$ & 35 (97.2) & 0.1954 & $97(80.8)$ & $22(81.5)$ & 0.767 \\
\hline $86 N / Y$ & $9(7.1)$ & $0(0.0)$ & & $5(4.2)$ & $2(7.4)$ & \\
\hline $86 Y$ & $14(11.1)$ & $1(2.8)$ & & $18(15.0)$ & $3(11.1)$ & \\
\hline $\begin{array}{l}\text { Pfmdr1 codon } \\
184\end{array}$ & $n=144$ & $n=36$ & & $n=140$ & $n=26$ & \\
\hline Y184 & $44(30.6)$ & $7(19.4)$ & 0.1029 & $38(27.1)$ & $5(19.2)$ & 0.464 \\
\hline $184 \mathrm{Y} / \mathrm{F}$ & 19 (13.2) & $0(0.0)$ & & 15 (10.7) & $3(11.5)$ & \\
\hline $184 \mathrm{~F}$ & $81(56.3)$ & $29(80.6)$ & & $87(62.1)$ & $18(69.2)$ & \\
\hline $\begin{array}{l}\text { Pfmdr1 codon } \\
1246\end{array}$ & $n=152$ & $n=39$ & & $n=146$ & $n=25$ & \\
\hline D1246 & 149 (98.0) & 39 (100.0) & 1 & 144 (98.6) & $24(96.0)$ & 1 \\
\hline 1246D/Y & $0(0.0)$ & $0(0.0)$ & & $0(0.0)$ & $1(4.0)$ & \\
\hline $1246 Y$ & $3(2.0)$ & $0(0.0)$ & & $2(1.4)$ & $0(0.0)$ & \\
\hline $\begin{array}{l}\text { Pfmdr1 } \\
\text { haplotypest }\end{array}$ & $n=124$ & $n=35$ & & $n=115$ & $n=24$ & \\
\hline NFD & $74(59.7)$ & $27(77.1)^{\star}$ & 0.014 & 71 (61.7) & $17(70.8)$ & 1 \\
\hline NYD & $56(45.2)$ & $7(20.0)$ & & 39 (33.9) & $6(25.0)$ & \\
\hline YFD & $20(16.1)$ & $1(2.9)$ & & 19 (16.5) & $4(16.7)$ & \\
\hline NFY & $0(0.0)$ & $0(0.0)$ & & $0(0.0)$ & $1(4.2)$ & \\
\hline NYY & $0(0.0)$ & $0(0.0)$ & & $0(0.0)$ & $1(4.2)$ & \\
\hline YYD & $4(3.2)$ & $0(0.0)$ & & $2(1.7)$ & $1(4.2)$ & \\
\hline YFY & $1(0.8)$ & $0(0.0)$ & & $0(0.0)$ & $1(4.2)$ & \\
\hline YYY & $2(1.6)$ & $0(0.0)$ & & $2(1.7)$ & $1(4.2)$ & \\
\hline
\end{tabular}

Bold letter denotes an encoded amino acid change 
† Haplotypes defined at codons 86,184 , and 1246; haplotype numerators include isolates with mixed infections

*Comparing wild-type vs. mutant (excluding mixed infections) for point mutations and NFD versus sum of non-NFD for haplotypes

Table 5: Prevalence of Pfcrt polymorphisms in paired pre-treatment and post-treatment samples in the artesunate-amodiaquine treatment arm, therapeutic efficacy monitoring, Mali, 2015-2016. 


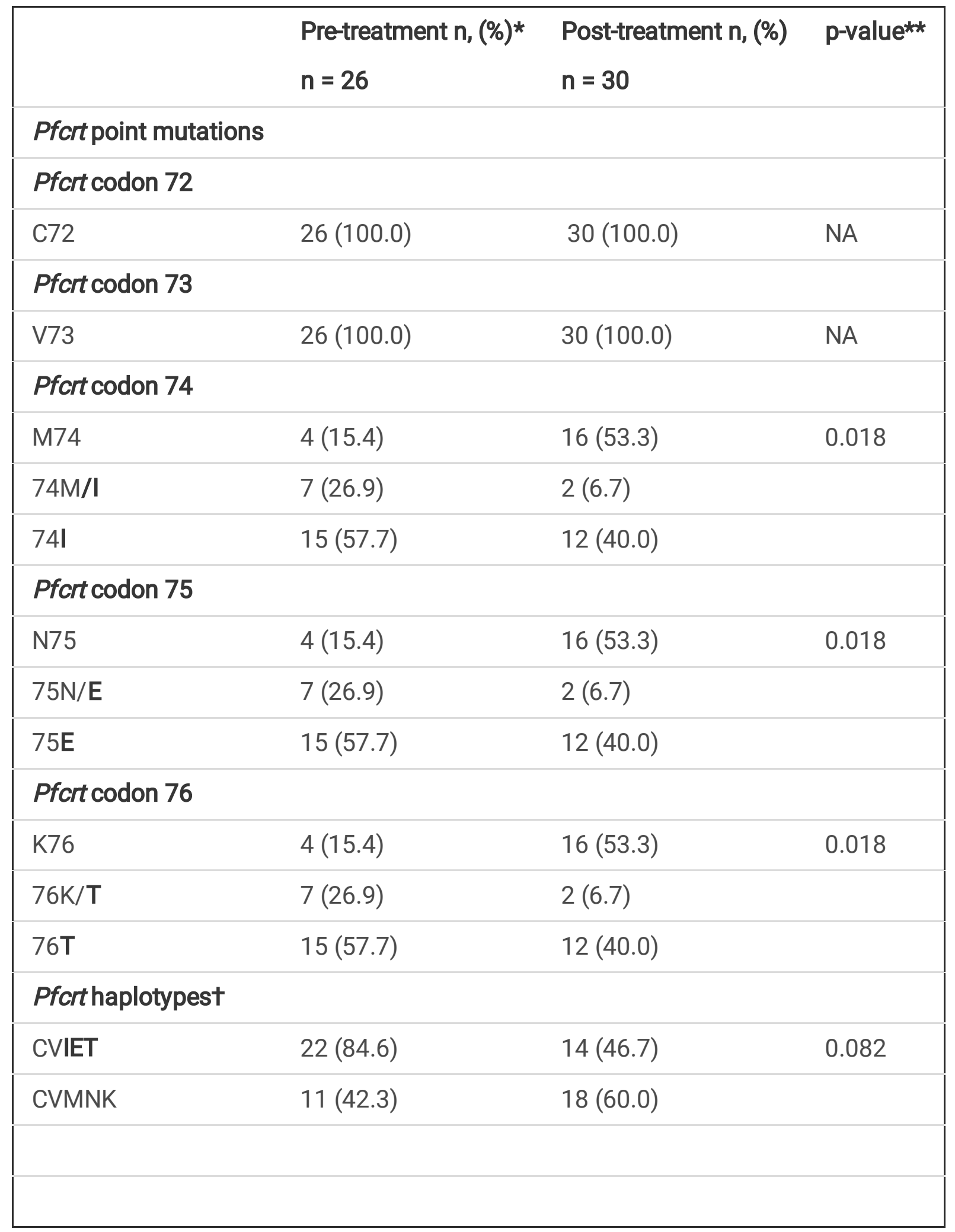

Bold letter denotes an encoded amino acid change; † Haplotype totals include mixed infections.

*4 recrudescent infections not included

** Comparing wild-type vs. mutant (excluding mixed infections) for point mutations and CVIET versus CVMNK haplotypes 
Figures

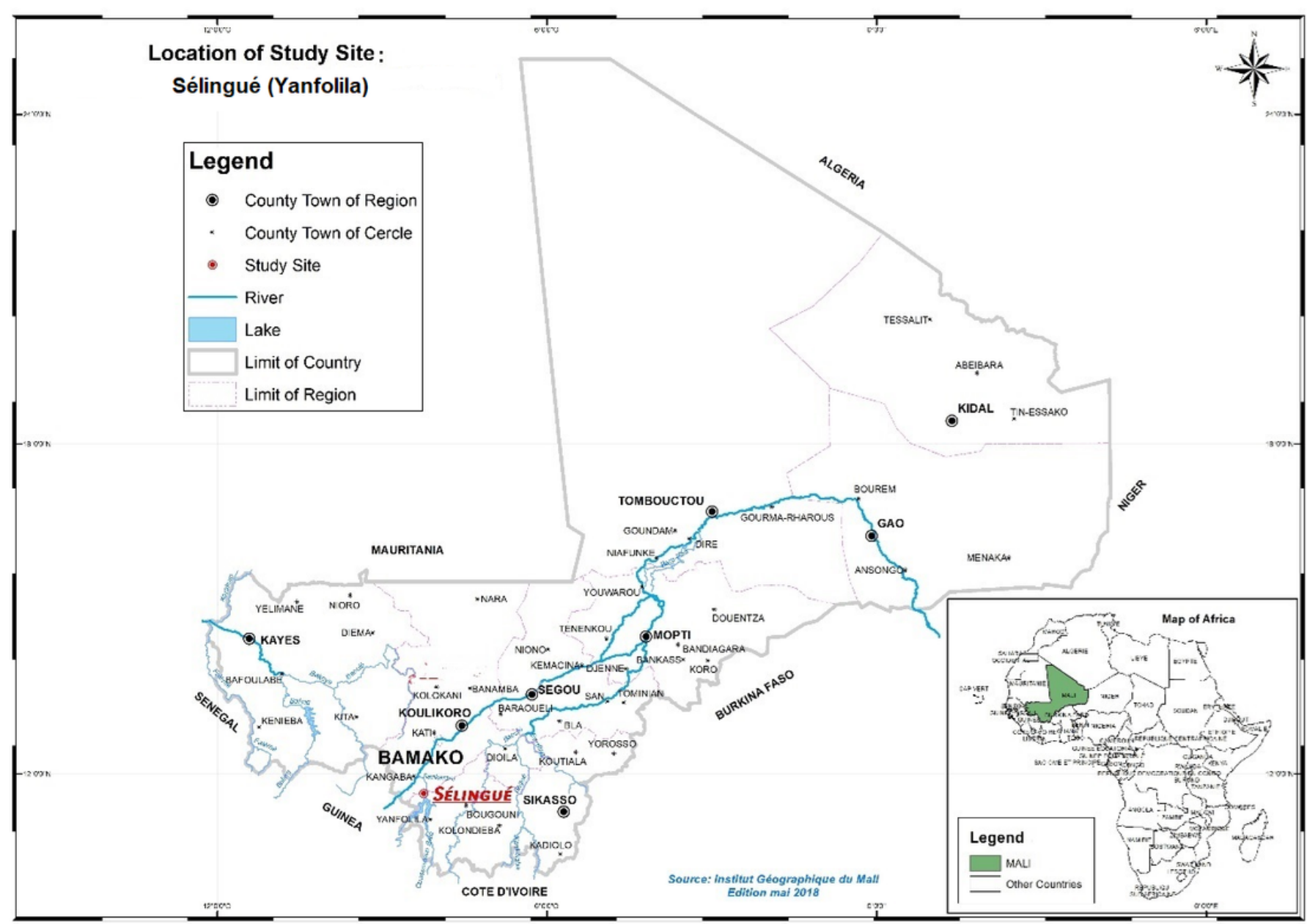

\section{Figure 1}

The Sélingué study site (in red font) used in the Mali therapeutic efficacy study, 2015-2016 (Institut National Geographique du Mali, May 2018). 
a.)Uncorrected

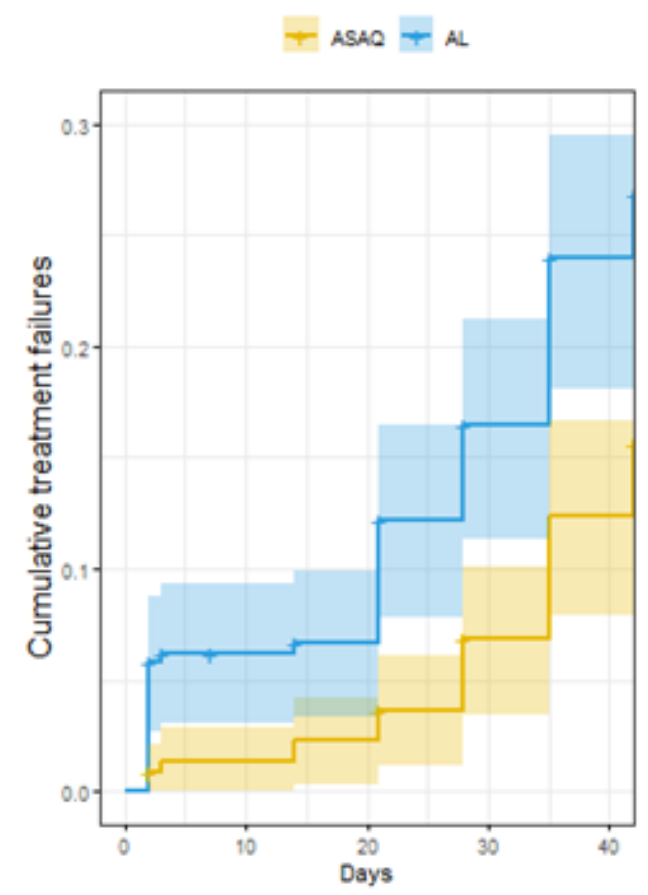

b.)PCR-corrected

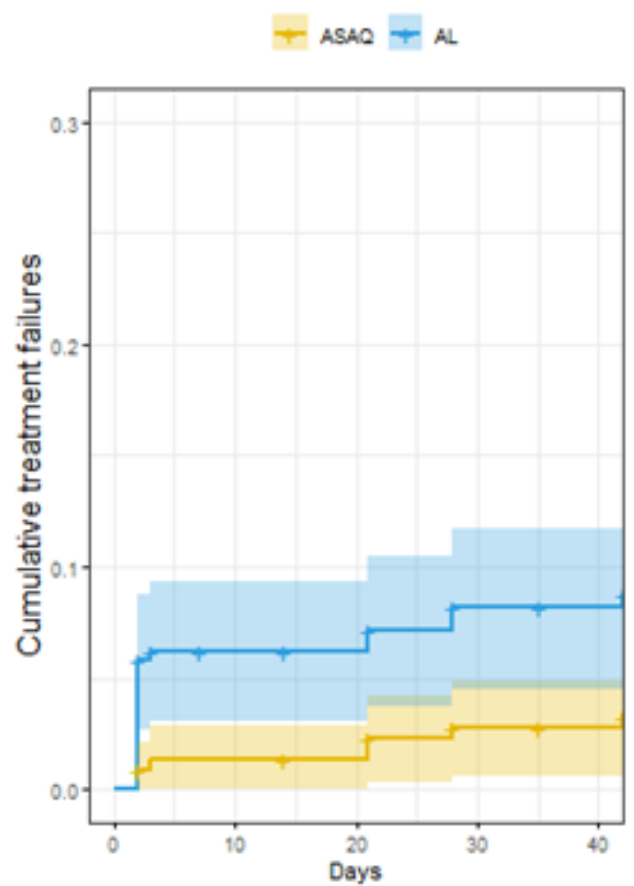

c.)Reinfection

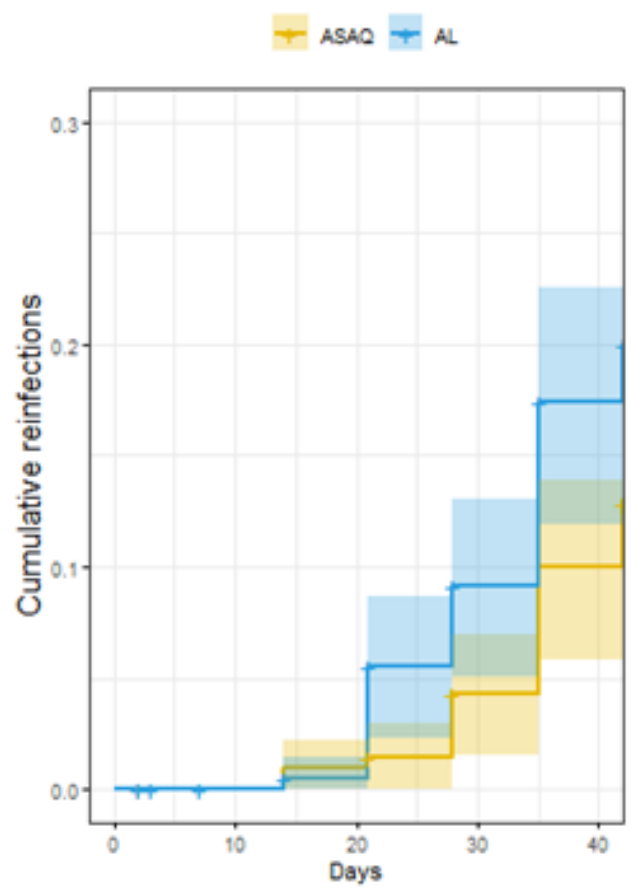

ASAQ: artesunate-amodiaquine

$\mathrm{AL}$ : artemether-lumefantrine

\section{Figure 2}

2a, 2b, and 2c: Kaplan Meier graphs of rates of: a) uncorrected treatment failures, b) recrudescent infections (PCR-corrected), and c) reinfections during 42 days of therapeutic efficacy monitoring for two ACTs in Sélingué, Mali, 2015-2016.

\section{Supplementary Files}

This is a list of supplementary files associated with this preprint. Click to download.

- SupplementalTable1TES2016MJ.doc 